\title{
Long-term outcomes of the aphakic snap-on Boston type I keratoprosthesis at the Bascom Palmer Eye Institute
}

This article was published in the following Dove Press journal:

Clinical Ophthalmology

\author{
Allister Gibbons' \\ Ella H Leung' \\ Luis J Haddock' \\ Carlos A Medina' \\ Viviana Fernandez ${ }^{2}$ \\ Jean-Marie A Parel ${ }^{1,2}$ \\ Heather A Durkee ${ }^{2}$ \\ Guillermo Amescua' \\ Eduardo C Alfonso' \\ Victor L Perez ${ }^{1,3}$
}

'Bascom Palmer Eye Institute/ University of Miami Miller School of Medicine, Miami, FL, ${ }^{2}$ Ophthalmic Biophysics Center, Bascom Palmer Eye Institute, University of Miami Miller School of Medicine, Miami, FL, ${ }^{3}$ Ocular Surface Center, Bascom Palmer Eye Institute, University of Miami Miller School of Medicine, Miami, FL, USA
Correspondence: Victor L Perez Department of Ophthalmology, Microbiology and Immunology, University of Miami Miller School of Medicine/Bascom Palmer Eye Institute, William McKnight Research Building, 1638 NW I0th Avenue, \#6I3, Miami, FL 33136, USA

$\mathrm{Tel}+\mathrm{I} 3053266302$

$\mathrm{Fax}+\mathrm{I} 3054824853$

Email vperez4@med.miami.edu
Purpose: To determine the indications, long-term clinical and visual outcomes, and complications of the aphakic snap-on type I Boston keratoprosthesis (KPro).

Design: Retrospective, non-comparative case series.

Methods: Forty-five eyes of 43 patients with type I aphakic snap-on KPros with at least 1 year of follow-up were included. The past medical histories, preoperative indications, best-corrected visual acuities (BCVAs), postoperative complications, and retention rates were analyzed.

Results: The most common indication for KPro implantation was a failed corneal graft (89\%). The mean preoperative BCVA was count fingers-hand motion $(2.14 \pm 0.45$ logarithm of minimum angle of resolution [ $\log$ MAR]), which initially improved to 20/200 (1.04 $\pm 0.85 \log$ MAR; $P<0.0001)$. At the last examination, 24 eyes (53\%) maintained some visual gain, $22 \%$ retained their preoperative visual acuity, and $24 \%$ lost vision due to postoperative events and underlying ocular comorbidities. Postoperative complications included retroprosthetic membranes $(8 / 45$, $18 \%)$, corneal melts $(5 / 45,11 \%)$, glaucoma progression $(6 / 45,13 \%)$, and endophthalmitis or sterile vitritis $(6 / 45,13 \%)$. The KPro retention rate was $89 \%$, with a mean follow-up of 51 months. The mean BCVA at the last visit was 20/1,400 (1.82 $\pm 0.92 \log M A R)$.

Conclusion: Most patients experienced improved visual acuity after the implantation of the aphakic, snap-on type I KPro; however, the visual gains were not sustained over time, correlating with the onset of postoperative complications.

Keywords: keratoprosthesis, corneal blindness, aphakia, penetrating keratoplasty, snap-on type I keratoprosthesis

\section{Plain language summary}

Eyes that underwent implantation of aphakic, snap-on type I keratoprostheses were likely to experience an improvement in vision; however, the visual gain decreased over time as patients developed postoperative complications.

\section{Introduction}

Several artificial corneas have been designed over the years, including the DohlmanDoane Boston Keratoprosthesis (KPro) (Massachusetts Eye and Ear Infirmary, Boston, MA, USA). ${ }^{1}$ Improvements in the device design, surgical techniques, and postoperative care have improved the clinical outcomes. ${ }^{2,3}$ Some of the changes include the use of hydrophilic methafilcon-A hydrogel or silicone-hydrogel bandage contact lenses to decrease desiccation and ulceration; prophylactic antimicrobials to prevent infections; placement of 16 holes in the back plate to maximize corneal nutrition; introduction of a threadless stem to minimize damage to the corneal graft during assembly; 
and addition of a titanium locking ring to guard against accidental unlocking. ${ }^{4}$

Most published series have combined several KPro models, including threaded or snap-on and pseudophakic and aphakic versions, making it difficult to draw comparisons of clinical outcomes. Aphakic KPros have been associated with better refractive outcomes than pseudophakic KPros. ${ }^{5}$ The purpose of the study is to determine the long-term clinical outcomes of eyes with snap-on aphakic Boston type I KPros implanted at the Bascom Palmer Eye Institute.

\section{Methods}

The retrospective case series was approved by the Institutional Review Board of the University of Miami. The patient data were collected and maintained in accordance with the Health Insurance Portability and Accountability Act guidelines. Patients signed a consent form for their records to be reviewed at the initiation of treatment. The study adhered to the tenants of the Declaration of Helsinki.

The medical records of 128 patients who underwent KPro implantation at the Bascom Palmer Eye Institute from 1980 to 2013 were reviewed. The decision to proceed with a KPro was made after extensive discussions with the patients, including the risks, benefits, and alternatives. The majority (89\%) had previously failed an average of 2.5 corneal transplants, and the chances of maintaining visual improvement after repeat grafts were less than those with KPros. ${ }^{6}$ Limbal stem cell transplants alone were deemed insufficient to treat the extent of ocular pathology. A KPro was, therefore, considered the most likely option to improve visual acuity. The inclusion criteria included snap-on aphakic Boston type I KPro. The exclusion criteria included children, patients with $<1$ year of follow-up, and incomplete medical records. Children were excluded due to the inability to perform accurate visual acuity testing. The Snellen visual acuity was converted to its logarithm of minimal angle of resolution (logMAR) equivalent as previously described; count-fingers was assigned a logMAR value of 1.9, hand motion 2.3, light perception 2.7 , and no light perception $3.0 .^{7}$

All surgeries occurred between 2007 and 2013. All patients were aphakic or were rendered so during KPro implantation. Additional procedures were performed at the surgeons' discretion (VLP, GA, ECA) based on the individual patient's clinical presentation. After the initial postoperative month, patients were examined every 2-4 months depending on their clinical course. At each visit, the conjunctival fornix was irrigated with $5 \%$ povidone-iodine, the large bandage contact lens was exchanged, and the topical antibiotics were rotated. ${ }^{3}$ All patients were started on topical prednisolone $1 \%$ and a fourth-generation fluoroquinolone 4-6 times a day postoperatively. Topical vancomycin $(25 \mathrm{mg} / \mathrm{mL})$ was concurrently begun in $47 \%$ of patients at the physician's discretion. A total of 36 eyes (80\%) used extended-wear bandage contact lenses (Kontur Kontact Lens Co., Hercules, CA, USA); 9 patients could not tolerate the contact lenses due to irritation and discomfort.

The information was compiled into several databases, and Kaplan-Meier survival curves, Fisher's exact test, and log-rank test were used to calculate statistical significance; a $P$-value $<0.05$ was considered statistically significant. The incidences of postoperative complications were calculated as cumulative incidences for each complication over the follow-up period to account for the varying lengths of follow-up for each patient.

\section{Results \\ Preoperative data}

A total of 45 eyes in 43 patients were included, with a mean follow-up of 51 months (range: 12-102 months). The indications for a KPro implantation included a previously failed graft $(40 / 45,89 \%)$, chronic ocular hypotony due to uveitis $(4 / 45,9 \%)$, and severe trauma $(1 / 45,2 \%)$. The underlying etiologies for the failed grafts included infectious keratitis, autoimmune keratitis, aniridia, and postsurgical complications (Table 1). Thirty-six eyes $(80 \%)$ had prior posterior segment disease, including previously repaired retinal detachments (RD, 5/45, 11\%), uveitis (4/45, 9\%), cystoid macular edema $(3 / 45,7 \%)$, previously treated endophthalmitis $(1 / 45,2 \%)$, and glaucoma $(27 / 45,60 \%)$. Due to the retrospective nature, the number of patients with limbal stem cell deficiency was unknown, but likely to be at least $8(18 \%)$. All eyes had preoperative best-corrected visual acuities (BCVAs) worse than 20/250 (average of count fingers-hand motion, $2.14 \pm 0.45 \log$ MAR).

\section{Intraoperative course}

A total of 42 eyes (93\%) had additional surgical procedures performed at the time of KPro implantation (Table 2), including lens removal surgery $(35 / 45,78 \%)$, iridectomy $(24 / 45,53 \%)$, glaucoma surgery $(17 / 45,38 \%)$, and vitrectomy $(38,84 \%)$. One patient (4\%) underwent cataract extraction, and $25(56 \%)$ had their intraocular lenses removed at the time of KPro implantation. Vitrectomies were performed to allow the insertion of pars plana glaucoma drainage implants and try to decrease the risk of retroprosthetic membranes. ${ }^{8}$ Unless the patient had an existing silicone oil tamponade, the vitreous was replaced with aqueous. One patient had an intraoperative serous choroidal detachment that resolved with observation postoperatively. 
Table I Demographical information, indications, and previously diagnosed comorbidities in patients receiving an aphakic Boston snap-on type I keratoprosthesis at the Bascom Palmer Eye Institute

\begin{tabular}{|c|c|}
\hline Ocular history & Group $(n=45)$ \\
\hline \multicolumn{2}{|l|}{ Demographics } \\
\hline Number of eyes & 45 \\
\hline Mean age at surgery (years $\pm S D$ ) & $66.7 \pm 16.8$ \\
\hline Females, n (\%) & $23(5 \mathrm{I})$ \\
\hline Right eye, n (\%) & $28(62)$ \\
\hline \multicolumn{2}{|l|}{ Prior corneal grafts } \\
\hline Prior failed graft, n (\%) & $40(89)$ \\
\hline Number of previously failed grafts, $n \pm S D$ & $2.5 \pm 1.0$ \\
\hline \multicolumn{2}{|l|}{ Underlying corneal pathology ${ }^{\mathrm{a}}$} \\
\hline Glaucoma related, n (\%) & II (24) \\
\hline Aphakic or pseudophakic bullous keratopathy, n (\%) & $7(16)$ \\
\hline Thermal/chemical burn, $\mathrm{n}(\%)$ & $4(9)$ \\
\hline Herpetic keratitis, n (\%) & $4(9)$ \\
\hline Uveitis, n (\%) & $4(9)$ \\
\hline Keratoconus, n (\%) & $2(4)$ \\
\hline Fuch's endothelial dystrophy, n (\%) & $2(4)$ \\
\hline Stevens-Johnson syndrome, $\mathrm{n}(\%)$ & $2(4)$ \\
\hline Retinal detachment related, $\mathrm{n}(\%)$ & $2(4)$ \\
\hline Aniridia, n (\%) & $\mathrm{I}(2)$ \\
\hline Corneal ulcer, n (\%) & I (2) \\
\hline Corneal exposure, $\mathrm{n}(\%)$ & $\mathrm{I}(2)$ \\
\hline Endophthalmitis, n (\%) & $I(2)$ \\
\hline Irido-corneal-endothelial syndrome, $\mathrm{n}(\%)$ & I (2) \\
\hline Mooren's ulcer, n (\%) & $\mathrm{I}(2)$ \\
\hline Riley day syndrome, n (\%) & $I(2)$ \\
\hline \multicolumn{2}{|l|}{ Contralateral eye } \\
\hline No significant pathology, n (\%) & $6(13)$ \\
\hline Visually impaired/legal blindness, n (\%) & $26(58)$ \\
\hline Blind/anophthalmic, n (\%) & $13(29)$ \\
\hline \multicolumn{2}{|l|}{ Preoperative lens status } \\
\hline Pseudophakia, n (\%) & $25(56)$ \\
\hline Aphakia, n (\%) & $19(42)$ \\
\hline \multicolumn{2}{|l|}{ Iris status } \\
\hline Aniridia, n (\%) & $14(31)$ \\
\hline \multicolumn{2}{|l|}{ Ocular and surgical history } \\
\hline Underlying glaucoma, any type, n (\%) & $34(76)$ \\
\hline Prior glaucoma drainage implant, $n$ (\%) & $25(26)$ \\
\hline Previous vitrectomy, $\mathrm{n}(\%)$ & $12(27)$ \\
\hline
\end{tabular}

Note: an patients with multiple ocular comorbidities, the most likely primary etiology leading to the need for KPro implantation was listed.

Abbreviations: Kpro, keratoprosthesis; n, number.

Table 2 Concomitant surgeries: concurrent surgical procedures performed at the time of implantation of the aphakic Boston snap-on type I keratoprosthesis

\begin{tabular}{ll}
\hline Concomitant surgeries & $\mathbf{n = 4 5}$ \\
\hline Vitrectomy & \\
Anterior vitrectomy, $\mathrm{n}(\%)$ & $18(40)$ \\
Pars plana vitrectomy, $\mathrm{n}(\%)$ & $20(44)$ \\
Previous PPV without repeat PPV at KPro implantation, $\mathrm{n}(\%)$ & $7(16)$ \\
Glaucoma & \\
Ahmed drainage implant, $\mathrm{n}(\%)$ & $9(20)$ \\
Molteno drainage implant, $\mathrm{n}(\%)$ & $6(13)$ \\
Endocyclophotocoagulation, $\mathrm{n}(\%)$ & $2(5)$ \\
\hline
\end{tabular}

Abbreviations: Kpro, keratoprosthesis; $n$, number; PPV, pars plana vitrectomy.

\section{Postoperative course}

Most eyes (39/45, 87\%) experienced an initial visual improvement. Five eyes (11\%) did not due to preexisting glaucoma, trauma, RD, or hypotony. One patient with endstage glaucoma reported worse vision in the immediate postoperative period.

Twenty-eight eyes (62\%) achieved 20/200 or better within 1 month of surgery (Figure 1A). After 1 year, however, only $64 \%$ retained vision better than $20 / 200$, and at 2 years, only $58 \%$ did. The mean visual acuity at the last follow-up was $20 / 1,400(1.82 \pm 0.92 \log$ MAR). Although the vision had significantly improved postoperatively $(P<0.0001)$, there was no statistically significant difference between the initial and final visual acuities $(P=0.43)$. The loss of initial visual acuity gain over time (Figure 1B) correlated with the onset of postoperative complications (Figure 1C), especially posterior segment pathologies (Figure 1D).

The cumulative incidence of postoperative complications during the follow-up period was 58\% (26/45). If only major sight-threatening complications were included, such as endophthalmitis, RD, corneal melts, Kpro extrusions, or glaucoma progression, then the incidence was $47 \%$ (21/45). The mean onset of the first postoperative complication was $22 \pm 8$ months. Anterior segment complications began at a mean of $20 \pm 12$ months, posterior segment complications at $21 \pm 17$ months, and sight-threatening complications at $28 \pm 9$ months.

Visually significant retroprosthetic membranes (RPMs) requiring surgical or laser intervention occurred in 8 eyes (18\%) (Figure 2A) and was the earliest postoperative complication to present, at a mean of $9 \pm 7$ months. Four eyes with recurrent RPMs after Yttrium Aluminum Garnet laser required pars plana surgical membranectomy. Corneal melts occurred in 5 eyes (11\%), occurring at a mean of $28 \pm 8$ months (Figure 2B). At the final follow-up visit, the retention rate of the initial KPro was $89 \%$ (40/45, Figure 2C).

The 6 eyes (13\%) that developed vision loss attributed to glaucoma had preexisting glaucoma (Figure 2D); 5 of these eyes had anterior chamber tubes that were considered functional at the time of KPro implantation. Four eyes $(8 \%)$ required a tube revision or removal during follow-up. Vision loss attributed to glaucoma was recorded at a mean of $33 \pm 21$ months.

Thirteen eyes $(27 \%)$ developed one or more posterior segment complications (Figure 2D). These included endophthalmitis or sterile vitritis, RD, vitreous hemorrhages, and an epiretinal membrane (ERM).

Six eyes (13\%) developed endophthalmitis or sterile vitritis (Figure 2E) at a mean of $27 \pm 19$ months. Two patients 

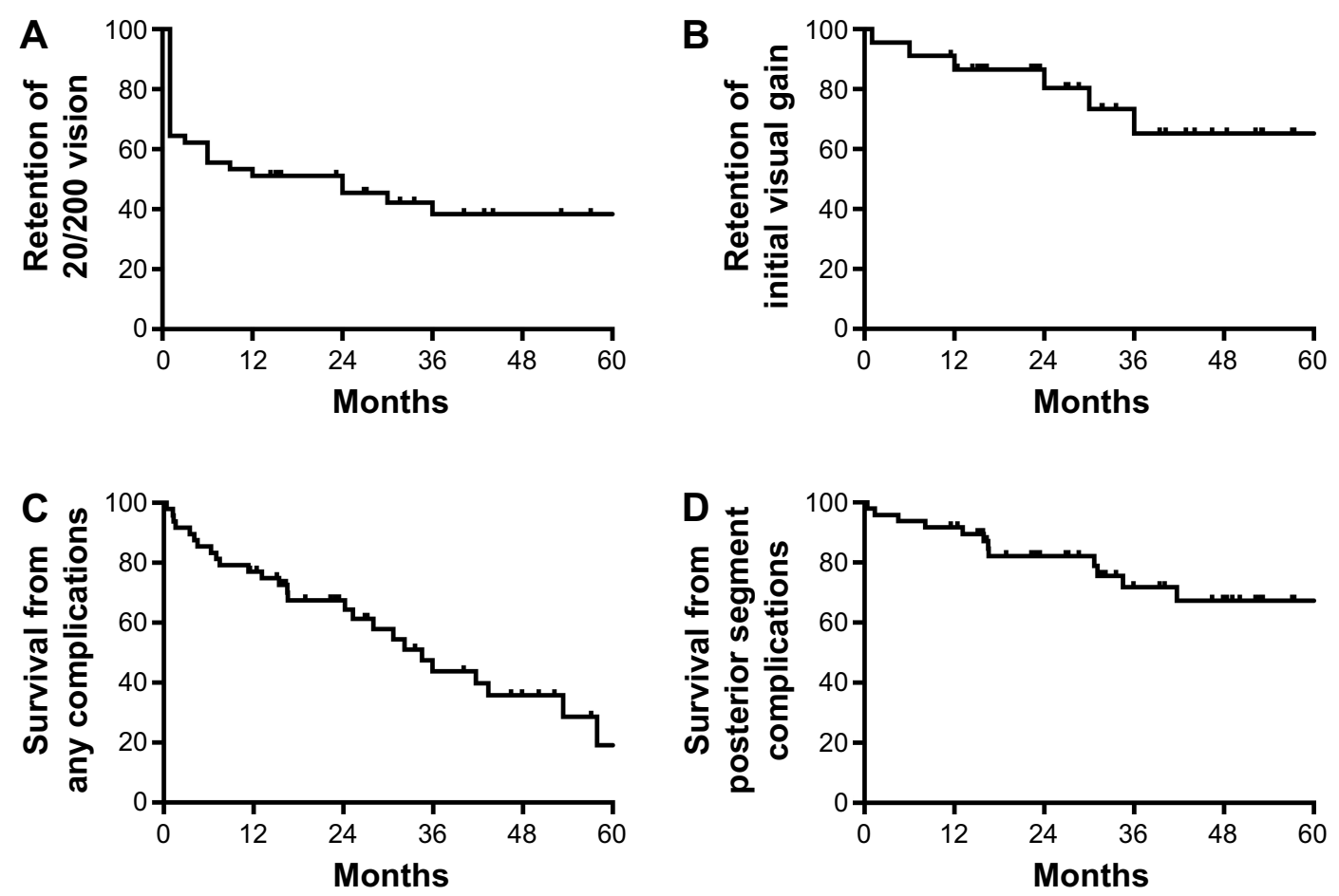

Figure I Kaplan-Meier survival graph of vision in patients receiving a Boston aphakic snap-on type I keratoprosthesis for the first 5 years of follow-up. Notes: (A) Retention of 20/200 vision over the follow-up period. (B) Maintenance of the initial visual gain over time. (C) Survival from any complication. (D) Survival from any posterior segment complication (retinal detachment, endophthalmitis, and epiretinal membranes).

were not using their prophylactic antibiotics, 3 patients had chronic leakage from their glaucoma drainage implants or KPros, and 1 patient was not using his bandage contact lens. Two patients ultimately had their KPros removed. The 2 eyes with positive vitreous cultures (Serratia marcescens and methicillin-sensitive Staphylococcus aureus) had final visual acuities of light perception and no light perception. The 3 eyes that retained their initial visual acuities had negative cultures.

Four patients (9\%) developed RD during the postoperative period (Figure $2 \mathrm{~F}$ ), occurring at a mean of $11 \pm 12$ months postoperatively. The vision regressed to the preoperative visual acuity or worse in these eyes. Two eyes (4\%) that developed vitreous hemorrhages postoperatively improved with observation, and 1 eye (2\%) developed an ERM that was subsequently removed.

\section{Discussion}

Corneal transplants are the treatment of choice for most patients with corneal blindness; however, repeat corneal transplants have lower success rates than initial transplants. ${ }^{9}$ More than $80 \%$ of repeat grafts fail within 5 years, and the length of graft survival and the best achieved visual acuity decreases with each failed graft. ${ }^{9}{ }^{, 10}$ In addition, patients with extensive corneal neovascularization, advanced glaucoma, and prior glaucoma filtering procedures are at higher risk of corneal graft failure. ${ }^{11}$ In these patients, a Boston KPro is a viable alternative to traditional donor corneal transplants. ${ }^{12}$ The number of implanted KPros continues to rise, and in 2014, there were 294 type I KPros implanted in the USA alone. ${ }^{13}$

A total of $87 \%$ of eyes in the present study experienced some improvement in their visual acuity after KPro implantation (Figure 3), comparable to the previously reported rates of $77 \%-84 \% .{ }^{2,14}$ Moreover, 28 eyes $(62 \%)$ were able to achieve a visual acuity of 20/200 or better during follow-up, which was a significant visual gain in eyes that have had multiple previous surgeries. The vision was comparable to the 20/200 attained in a large multicenter study of 300 KPro eyes followed for 17 months. ${ }^{15}$ During the extended 51-month follow-up of the current study, however, the vision slowly declined, corresponding to the onset of postoperative complications (Figures 2 and 3). A recently published study noted a similar trend; in eyes that achieved a visual acuity $\geq 20 / 200$ at 1 year of follow-up, only $59 \%$ maintained that vision at 2 years, $50 \%$ at 3 years, and $29 \%$ at 4 years. ${ }^{16-18}$ Although the improvement in BCVA was not long-lasting in many patients, the temporary gain likely had a positive impact on the patients' lives.

Retroprosthetic membranes were the most common postoperative complication (18\%), similar to previously published rates of $26 \%-65 \% .^{2,14}$ The exact etiology for 
A

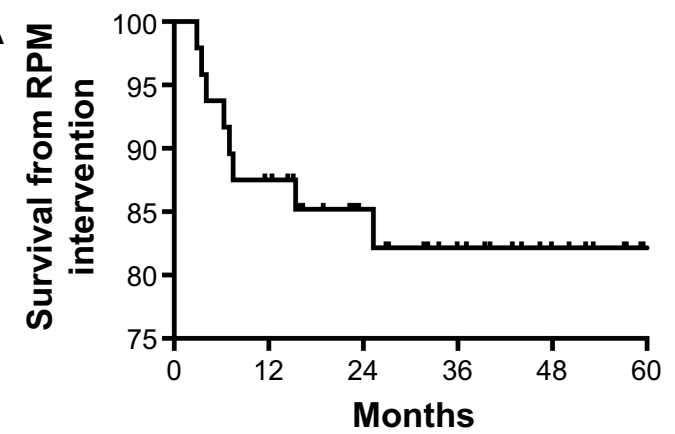

C

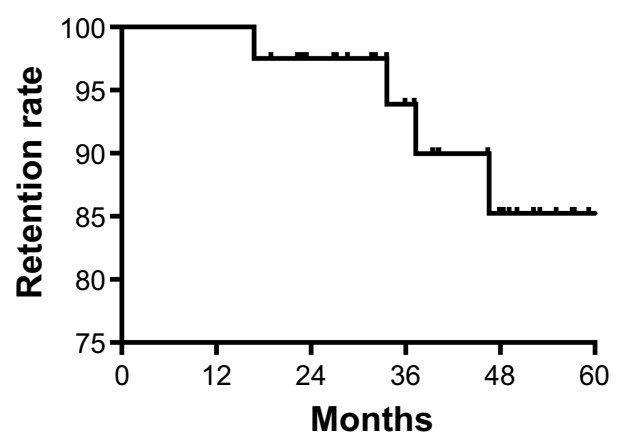

E

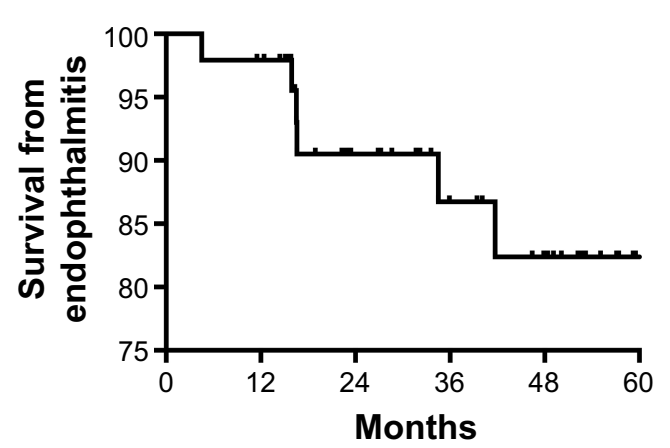

B

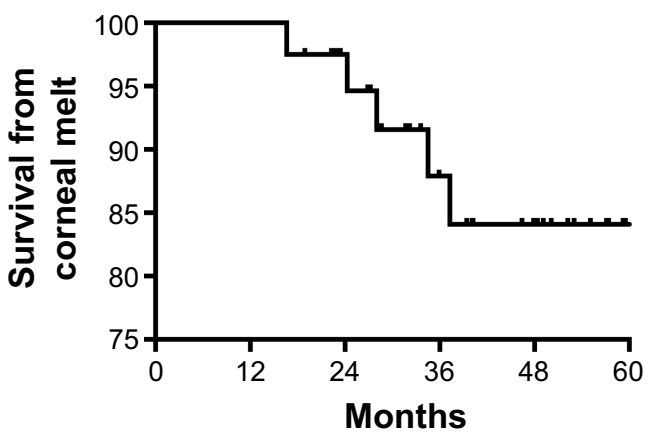

D

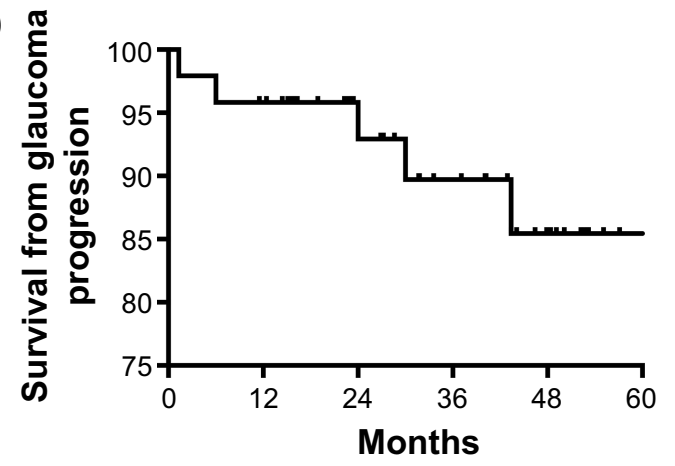

F

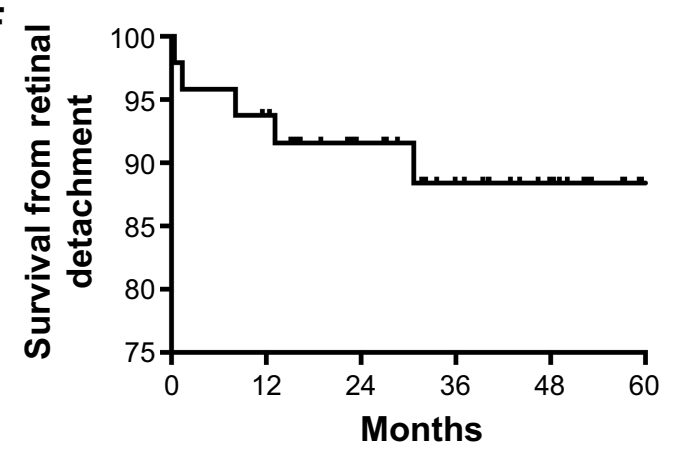

Figure 2 Kaplan-Meier survival graph of the most frequent complications in patients receiving an aphakic Boston snap-on keratoprosthesis during the first 5 years of follow-up. Notes: (A) Retroprosthetic membranes that required intervention during the follow-up period. (B) Survival from corneal melts. (C) Survival from keratoprosthesis extrusion or removal (retention). (D) Survival from loss of vision attributed to glaucoma progression. (E) Survival from endophthalmitis or sterile vitritis. (F) Survival from retinal detachments.

Abbreviation: RPM, retroprosthetic membrane.

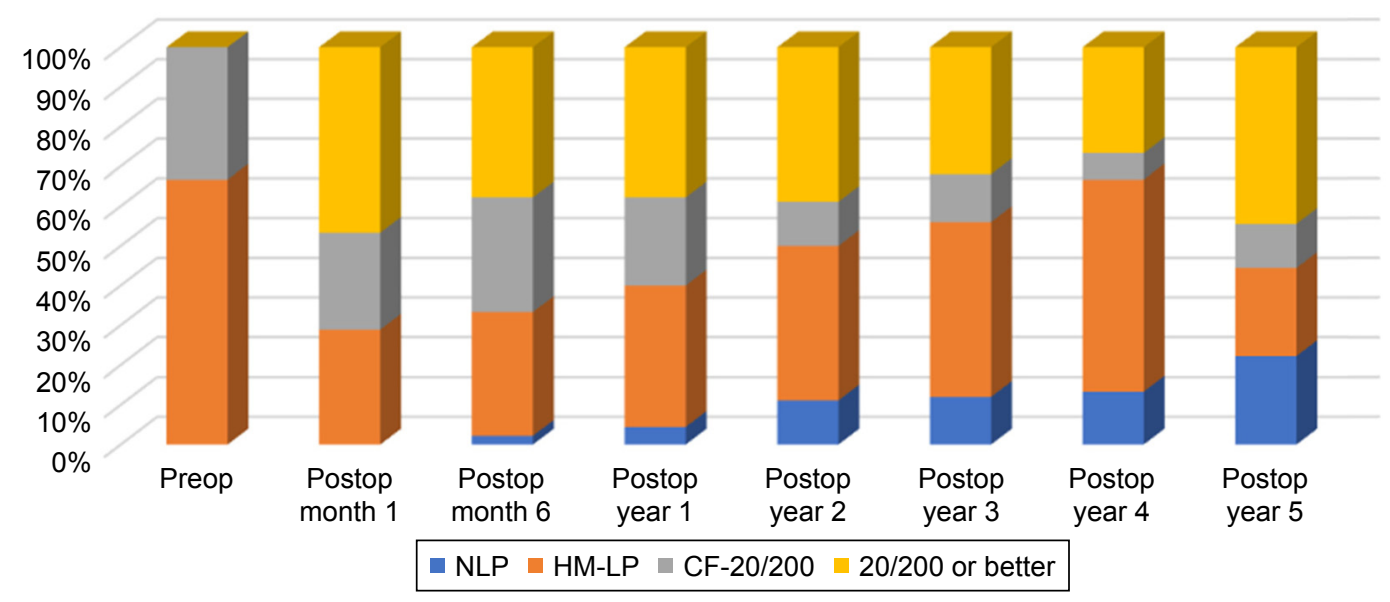

Figure 3 Visual acuities over time.

Note: The visual acuities improved initially postoperatively, then gradually declined over time.

Abbreviations: CF, count fingers; HM, hand motion; LP, light perception; NLP, no light perception; Preop, preoperatively; Postop, postoperatively. 
RPM formation is unclear, but an inflammatory reaction and cellular downgrowth have been suggested. Histopathological studies of RPMs have found inflammatory cytokines, as well as stromal downgrowth from the host's cornea, host's iris stroma, and retro-iridial areas. ${ }^{19,20}$

RPM formation has been linked to the development of other complications, such as corneal melts and extrusions. ${ }^{21,22}$ The incidence of tissue melts and KPro extrusions was similar between our study and other series with similar follow-up periods (11\% versus $1 \%-29 \%$, respectively). ${ }^{23}$ Our retention rate at the last follow-up was of $89 \%$, similar to the $83 \%-97 \%$ previously reported for others types of Boston type I KPros in medium- to long-term studies. ${ }^{16-18}$

Glaucoma was prevalent preoperatively (69\%). The majority $(90 \%)$ was still able to experience an initial improvement in the vision postoperatively; however, the visual gains were lost over time as the glaucoma progressed despite apparent adequate management, which has been previously described. ${ }^{24-28}$ The preexisting glaucoma drainage tubes were considered functional at the time of Kpro implantation so no additional glaucoma surgeries were performed at the time. In patients with underlying glaucoma, however, the surgeon may consider flushing, replacing, or adding a second glaucoma drainage implant at the time of KPro surgery to better control the intraocular pressures. Perhaps implantable sensors that are capable of measuring intraocular IOPs in KPro eyes may mitigate vision loss from glaucoma in the future. ${ }^{29-31}$ No significant association was found between glaucoma shunts and the development of endophthalmitis or KPro extrusions in our series.

The rate of endophthalmitis or vitritis in our study (13\%) was similar to those of other large previously published series $(0 \%-11 \%) .^{2,14,32,33}$ Risk factors identified in the present series included no prophylactic antibiotics, no bandage contact lenses, and leakage from the glaucoma drainage implants or KPros. The two patients with culture-positive endophthalmitis had worse visual acuities than those with culture-negative vitritis. Sterile vitritis has been previously described in eyes with KPros, usually presenting with painless vision loss, negative cultures, good response to periocular steroids, and good final visual acuities after treatment. ${ }^{34}$ All patients in the current series were treated for presumed infectious endophthalmitis given the potentially devastating consequences of infections and the inherent risks of implanted intraocular hardware.

The incidences of RD (9\%) and ERMs (2\%) were similar to those of other studies $(0 \%-19 \%$ for RD and $1 \%-3 \%$ for ERM). ${ }^{2,14,32,35}$ All eyes had successful repair of their RD, but only 1 patient regained the pre-detachment vision.
The patients in the current series had significant ocular comorbidities and had multiple prior intraocular surgeries. Even without surgical intervention, these eyes had poor visual prognoses. The 6 patients whose vision did not improve postoperatively had preexisting conditions that limited their potential visual gain; none were due to intraoperative complications. As certain sight-limiting conditions like macular scars or optic neuropathies may not be visible on traditional ultrasounds, preoperative electrophysiologic studies and endoscopic evaluations may help determine whether preexisting comorbidities may limit the potential visual acuity of KPro candidates. ${ }^{36}$

The limitations of the present study include its retrospective nature, small sample size, variability in patient follow-up, and prolonged study period. Nevertheless, the study size was similar to those of other single-center KPro studies. Furthermore, the mean follow-up period was 51 months, allowing for comparison of early and late postoperative clinical outcomes.

In conclusion, the visual acuity outcomes and rates of postoperative complications at our institution with aphakic KPros were similar to those of other tertiary care facilities with pseudophakic and aphakic KPros. ${ }^{2,5,14,32,35}$ The vision initially improved, but it declined over time as patients developed postoperative complications, including glaucoma progression, endophthalmitis, and RD. The results underline the importance of adhering to the current prophylactic antibiotic recommendations and of continuing to improve the KPro design, surgical techniques, and postoperative management to avoid potential sight-threatening postoperative complications.

\section{Acknowledgments}

We are indebted to Tayyeba K Ali, MD, for her knowledge, hard work, and guidance during the course of the research. This research was supported by the Florida Lions Eye Bank, NEI Core Grant (EY0,14,801), Research to Prevent Blindness Unrestricted Grant, Department of Defense (DOD, Grant\# W81XWH-09-1-0675 and Grant\# W81XWH-13-10048 ONOVA) (institutional), the Henri and Flore Lesieur Foundation (JMP); and Walter G Ross Chair in Ophthalmic Research (VLP).

\section{Disclosure}

Dr Victor L Perez has served as a consultant, a member of the speaker's bureau, or both for Alcon, Bausch \& Lomb, EyeGate, and Shire. This does not pertain to the subject of this article. The other authors report no conflicts of interest in this work. 


\section{References}

1. Akpek EK, Alkharashi M, Hwang FS, Ng SM, Lindsley K. Artificial corneas versus donor corneas for repeat corneal transplants. Cochrane Database Syst Rev. 2014;11:CD009561.

2. Aldave AJ, Kamal KM, Vo RC, Yu F. The boston type I keratoprosthesis: improving outcomes and expanding indications. Ophthalmology. 2009;116(4):640-651.

3. Behlau I, Martin KV, Martin JN, et al. Infectious endophthalmitis in Boston keratoprosthesis: incidence and prevention. Acta Ophthalmol. 2014;92(7):e546-e555.

4. Gomaa A, Comyn O, Liu C. Keratoprostheses in clinical practice - a review. Clin Exp Ophthalmol. 2010;38(2):211-224

5. Utine CA, Tzu J, Dunlap K, Akpek EK. Visual and clinical outcomes of explantation versus preservation of the intraocular lens during keratoprosthesis implantation. J Cataract Refract Surg. 2011;37(9): 1615-1622.

6. Ahmad S, Mathews PM, Lindsley K, et al. Boston Type 1 Keratoprosthesis versus repeat donor keratoplasty for corneal graft failure: a systematic review and meta-analysis. Ophthalmology. 2016; 123(1):165-177.

7. Lange C, Feltgen N, Junker B, Schulze-Bonsel K, Bach M. Resolving the clinical acuity categories "hand motion" and "counting fingers" using the Freiburg Visual Acuity Test (FrACT). Graefes Arch Clin Exp Ophthalmol. 2009;247(1):137-142.

8. Perez VL, Leung EH, Berrocal AM, et al. Impact of total pars plana vitrectomy on postoperative complications in aphakic, snap-on, type 1 Boston keratoprosthesis. Ophthalmology. 2017;124(10):1504-1509.

9. Ma JJ, Graney JM, Dohlman CH. Repeat penetrating keratoplasty versus the Boston keratoprosthesis in graft failure. Int Ophthalmol Clin. 2005;45(4):49-59.

10. Bersudsky V, Blum-Hareuveni T, Rehany U, Rumelt S. The profile of repeated corneal transplantation. Ophthalmology. 2001;108(3): 461-469.

11. Chew HF, Ayres BD, Hammersmith KM, et al. Boston keratoprosthesis outcomes and complications. Cornea. 2009;28(9):989-996.

12. Ament JD, Stryjewski TP, Ciolino JB, Todani A, Chodosh J, Dohlman CH. Cost-effectiveness of the Boston keratoprosthesis. Am J Ophthalmol. 2010;149(2):221.e2-228.e2.

13. EBAA. 2014 Eye Banking Statistical Report. Washington, DC: Eye Bank Association of America. Available from: http://restoresight.org/ wp-content/uploads/2015/03/2014_Statistical_Report-FINAL.pdf. Accessed November 22, 2017.

14. Aldave AJ, Sangwan VS, Basu S, etal. International results with the Boston type I keratoprosthesis. Ophthalmology. 2012;119(8):1530-1538.

15. Rudnisky CJ, Belin MW, Guo R, Ciolino JB; Boston Type 1 Keratoprosthesis Study Group. Visual acuity outcomes of the Boston keratoprosthesis type 1: multicenter study results. Am J Ophthalmol. 2016; 162:89.e1-98.e1.

16. Greiner MA, Li JY, Mannis MJ. Longer-term vision outcomes and complications with the Boston type 1 keratoprosthesis at the University of California, Davis. Ophthalmology. 2011;118(8):1543-1550.

17. Mori Y, Nejima R, Minami K, Miyata K, Kamiya K, Fukud M. [Longterm outcomes of Boston keratoprosthesis]. Nippon Ganka Gakkai Zasshi. 2013;117(1):35-43.

Clinical Ophthalmology

\section{Publish your work in this journal}

Clinical Ophthalmology is an international, peer-reviewed journal covering all subspecialties within ophthalmology. Key topics include: Optometry; Visual science; Pharmacology and drug therapy in eye diseases; Basic Sciences; Primary and Secondary eye care; Patient Safety and Quality of Care Improvements. This journal is indexed on Submit your manuscript here: http://www.dovepress.com/clinical-ophthalmology-journal
18. Srikumaran D, Munoz B, Aldave AJ, et al. Long-term outcomes of Boston type 1 keratoprosthesis implantation: a retrospective multicenter cohort. Ophthalmology. 2014;121(11):2159-2164.

19. Stacy RC, Jakobiec FA, Michaud NA, Dohlman CH, Colby KA. Characterization of retrokeratoprosthetic membranes in the Boston type 1 keratoprosthesis. Arch Ophthalmol. 2011;129(3):310-316.

20. Hou JH, Sivaraman KR, de la Cruz J, Lin AY, Cortina MS. Histopathological and immunohistochemical analysis of melt-associated retroprosthetic membranes in the Boston type 1 keratoprosthesis. JAMA Ophthalmol. 2014;132(9):1133-1136.

21. Sivaraman KR, Hou JH, Allemann N, de la Cruz J, Cortina MS. Retroprosthetic membrane and risk of sterile keratolysis in patients with type I Boston keratoprosthesis. Am J Ophthalmol. 2013;155(5):814-822.

22. Robert MC, Dohlman $\mathrm{CH}$. A review of corneal melting after Boston keratoprosthesis. Semin Ophthalmo. 2014;29(5-6):349-357.

23. Yaghouti F, Nouri M, Abad JC, Power WJ, Doane MG, Dohlman $\mathrm{CH}$. Keratoprosthesis: preoperative prognostic categories. Cornea. 2001;20(1):19-23.

24. Kamyar R, Weizer JS, de Paula FH, et al. Glaucoma associated with Boston type I keratoprosthesis. Cornea. 2012;31(2):134-139.

25. Nguyen P, Chopra V. Glaucoma management in Boston keratoprosthesis type I recipients. Curr Opin Ophthalmol. 2014;25(2):134-140.

26. Crnej A, Paschalis EI, Salvador-Culla B, et al. Glaucoma progression and role of glaucoma surgery in patients with Boston keratoprosthesis. Cornea. 2014;33(4):349-354.

27. Banitt M. Evaluation and management of glaucoma after keratoprosthesis. Curr Opin Ophthalmol. 2011;22(2):133-136.

28. Panarelli JF, Ko A, Sidoti PA, Garcia JP, Banitt MR. Angle closure after Boston keratoprosthesis. J Glaucoma. 2013;22(9):725-729.

29. Wang D, Yu J, Tian L, Wang L, Huang Y. Effectiveness of transscleral cyclophotocoagulation as adjuvant therapy for refractory glaucoma in keratoprosthesis patients. Eur J Ophthalmol. 2015;25(1):8-13.

30. B3C Newswire. Implandata: first keratoprosthesis patient in Europe receives permanent $24 \mathrm{~h}$ intraocular pressure sensor eye mate [press release]. B3C Newswire; 2015.

31. Rivier D, Paula JS, Kim E, Dohlman CH, Grosskreutz CL. Glaucoma and keratoprosthesis surgery: role of adjunctive cyclophotocoagulation. J Glaucoma. 2009;18(4):321-324.

32. Lekhanont K, Thaweesit P, Muntham D, Chuckpaiwong V, Vongthongsri A. Medium-term outcomes of Boston type 1 keratoprosthesis implantation in Bangkok, Thailand. Cornea. 2014;33(12): 1312-1319.

33. Li JY, Greiner MA, Brandt JD, Lim MC, Mannis MJ. Long-term complications associated with glaucoma drainage devices and Boston keratoprosthesis. Am J Ophthalmol. 2011;152(2):209-218.

34. Grassi CM, Crnej A, Paschalis EI, Colby KA, Dohlman CH, Chodosh J. Idiopathic vitritis in the setting of Boston keratoprosthesis. Cornea. 2015;34(2):165-170.

35. Zerbe BL, Belin MW, Ciolino JB; Boston Type 1 Keratoprosthesis Study Group. Results from the multicenter Boston type 1 keratoprosthesis study. Ophthalmology. 2006;113(10):1779.e1-1779.e7.

36. Farias CC, Ozturk HE, Albini TA, et al. Use of intraocular video endoscopic examination in the preoperative evaluation of keratoprosthesis surgery to assess visual potential. Am J Ophthalmol. 2014;158(1): 80.e2-86.e2.

PubMed Central and CAS, and is the official journal of The Society of Clinical Ophthalmology (SCO). The manuscript management system is completely online and includes a very quick and fair peer-review system, which is all easy to use. Visit http://www.dovepress.com/ testimonials.php to read real quotes from published authors. 